\title{
A Comparison of Mixed Integer Programming Models for the Construction Site Layout Problem
}

\author{
A.W.A. Hammad ${ }^{a}$, A.Akbarnezhad ${ }^{a}$ and D. Rey $^{a}$ \\ ${ }^{a}$ School of Civil and Environmental Engineering, University of New South Wales, Australia \\ E-mail: a.hammad@unsw.edu.au, a.akbarnezhad@unsw.edu.au,d.rey@unsw.edu.au
}

\begin{abstract}
Site layout planning has been widely investigated in the literature as a means of minimising transportation costs, improving safety etc. The majority of the research conducted focuses on developing adequate formulations capable of producing optimum results. However, there is a lack of consensus on a particular format to implement for the problem, particularly in regards to the assumptions and constraints to be adopted. Distance between facilities, which is an important measure in site layout planning, has been modelled in several ways, all in the hope of producing reasonable approximations to the path followed by workers, machinery etc. between facilities. This paper aims to compare the performance of several models of the site layout problem. The objective function considered in this study is the transportation cost of material movement between facilities. A main mixed integer programming model is proposed to represent the site layout problem and a comparison is carried out against a relaxed variant, where travel distances are approximated. Further, a constraint generation algorithm is presented in a bid to improve the computational performance of the main model.

Keywords -

Construction site layout planning; Optimisation; Mixed Integer Programming;
\end{abstract}

\section{Introduction}

It is vital to carefully consider the site layout adopted on a construction site as performance and cost expended during construction can be directly affected by the location of different facilities on the site. [1]. The task of finding an appropriate allocation for construction facilities is dubbed as site layout planning (SLP). The SLP problem is an extensively investigated area in the construction research community, and interest in this subject has been constantly growing in the past decade or so with the drastic surge experienced in computer power and performance. The problem originally stems from the facility layout problem, developed in industrial operational research, where the goal is to minimise travel distances between departments labelled as facilities [2,3]. In the construction field, the problem comprises the allocation of work facilities to predefined spaces within a construction site, where an objective function is assigned to determine the direction of the optimisation [4,5]. In the literature, different objective functions have been embraced, ranging from functions that minimise safety hazards, minimise wildlife interference, minimise constraint violation and minimise construction noise levels, to the more common goal of minimising transportation and material handling costs [6-13]. Multiple objectives have also been implemented, where several of the aforementioned objectives are addressed all at once [14-19]. A look through the literature also reveals that the majority of problems are solved using metaheuristic techniques, where the reliance of these algorithms is more on random search [20-26]. This being said, there are instances however where the problem has been addressed using exact mathematical techniques [27,28]. Unlike stochastic search, mathematical programming allows for a global optimum solution to be reached. The only drawback is that, even though no formal proof yet exists, the construction SLP problem is thought to be NP-complete, and with computational solving times exceeding those that result from meta-heuristic techniques, adopting an exact method can result in computational inefficient solutions [29]. This can also mean that a more relaxed formulation might need to be adapted when solving such kind of problems.

For the material handling objective function, which will be the main focus of this paper, several approaches have been applied in the literature for estimating the distance travelled between two facilities on the construction site. These approaches rely on the approximation of the transportation route through the use of either the Euclidean or Manhattan distance measure. In the Euclidean method, the routes are taken to be diagonal lines extending from the centroids of the corresponding facilities between which movement is 
necessary [18,24,27,30,31]. The Manhattan approach relies on robotic movements in four directions at 90 degrees to one another [32,33]. The aforementioned approaches have their strengths and weaknesses but one thing common to both is their over-simplification of the actual route typical in a construction site, where obstacles such as facilities etc. might prevent a purely diagonal or rectilinear movement from being achieved. For this reason it is necessary to take into account such limitations when it comes to modelling distance measures. Therefore a model that tackles some of these issues is presented by the authors. A comparison will ensue to determine whether it's worthwhile to overburden the model with additional constraints to produce more realistic route mappings within the objective function. It is worth mentioning that the Euclidean approach seems much of a simplistic approximation as path obstruction and the possibility of passing through forbidden areas is completely ignored. Thus in this study the focus will mainly be on routes modelled via the Manhattan approach.

A computational assessment of SLP models is necessary to ensure that efficient solutions are being generated in reasonable amounts of time. In many of the studies reviewed on SLP the reported computational times of exact optimisation algorithms usually exceed what would normally be within an acceptable range. According to Smith-Miles and Lopes [34] a couple of the many determinants of the instance difficulties of combinatorial methods include the parameters implemented as well as the feasible area outlined through the constraints adopted within the mathematical model. The more constrained a problem is the more restricted is its feasible region. This can lead to difficulties for the optimisation algorithm, particularly when the feasible landscape is characterised by a nonconvex shape, as is the case in combinatorial optimisation problems [35]. To the authors' best knowledge no attempt has been made in the literature to analyse instance difficulty of the construction SLP problem.

This paper presents a comparison between several mixed integer programming (MIP) models for solving the SLP problem, where the objective is to minimise transportation costs. Contrasting several SLP model formulations, where distance is highlighted as the major area of focus, aims to shed light on the variance in computational efficiency between the models. To this avail the results presented in this paper may be helpful in the selection of an appropriate model, in terms of route mappings, for a particular site layout problem, based on solution optimality and computational performance.

\section{Models for the SLP}

Three different SLP models are presented in this section, namely Model F-SLP, O-SLP and R-SLP. The main model, F-SLP, is used as the benchmark model against which all other models are compared. For this model a full representation of route mapping is provided where a forbidden region, modelled as the construction area building footprint, is taken into consideration, such that distance is measured based on corner mapping to prevent unreasonable travel paths on the construction site. Design constraints, consisting of non-overlap constraints, boundary constraints and travel interference constraints, are introduced to define the model. Model O-SLP neglects the presence of a forbidden area and hence travel distance is assumed to be direct between facility centroids. For Model R-SLP, a constraint generation algorithm is introduced where the design constraints of Model F-SLP are first relaxed and then gradually added to the relaxed model (R-SLP) if they are violated. The problem is transformed so that the overall MIP model is broken down into smaller MIP models. To produce the MIP sub models in this relaxed model, any non-linearity present in the objective functions and/ or constraints is dealt with wherever possible through the adoption of common mathematical programming reformulations [36].

\subsection{Model Notation}

Tables 1-3 provide a list of the notations of sets, parameters and variables, respectively, adopted in the models. Any intermediate variables introduced later on in the paper which are not defined in the aforementioned tables will be detailed in the relevant sections.

\section{$2.2 \quad$ F-SLP: Forbidden Region SLP}

As was stated earlier, for the purpose of this study, Model F-SLP is taken to be the benchmark formulation, against which all the other presented models will be compared. The composition of this chief model consists of an objective function that tries to capture a realistic path planning approximation on construction sites, where obstacles such as facilities and construction building footprint area, are modelled and are taken into account as part of the constraints. Distance is assumed to be directly dependent on the exact position of the available spaces in which facilities are allocated, so that direct travel between adjacent locations is possible, whereas more manoeuvres need to be made between facilities located at far apart areas. The objective function along with the constraints, are presented below. 
Table 1 Set notation

\begin{tabular}{|c|c|}
\hline Notation & Description \\
\hline$F_{T}$ & $\begin{array}{l}\text { Set of all temporary facilities to be } \\
\text { allocated a position on-site }\end{array}$ \\
\hline$F_{P}$ & $\begin{array}{l}\text { Set of all permanent facilities with } \\
\text { predefined positions on-site }\end{array}$ \\
\hline$F: F_{T} \cup F_{P}$ & Set of all facilities on site \\
\hline$L$ & $\begin{array}{l}\text { Set of all available locations within } \\
\text { which temporary facilities will be }\end{array}$ \\
\hline$M$ & $\begin{array}{l}\text { Set of all non-available locations, } \\
\text { within which permanent facilities are }\end{array}$ \\
\hline$V=\left\{(i, m): \forall i \in F_{p}, \forall m \in M\right\}$ & $\begin{array}{l}\text { Mapping each permanent facility to its } \\
\text { pre-defined location }\end{array}$ \\
\hline$C_{m}$ & $\begin{array}{l}\text { Set of corners assigned to each location } \\
m \in L \cup M\end{array}$ \\
\hline
\end{tabular}

Table 2 Parameter notation

\begin{tabular}{|c|c|}
\hline Notation & Description \\
\hline$F_{i j t}$ & $\begin{array}{l}\text { Frequency of travel by transportation mode } t \text {, from facility } \\
i \text { to facility } j\end{array}$ \\
\hline$\delta_{m n}$ & $\begin{array}{l}\text { Binary parameter which equals one if two locations } m \text { and } \\
n \text { are deemed to be far from one another, and zero } \\
\text { otherwise. }\end{array}$ \\
\hline$\lambda_{i m}$ & $\begin{array}{l}\text { Binary parameter which equals one if facility } i \text { has a } \\
\text { predefined location } m \text { assigned to it, and zero otherwise. }\end{array}$ \\
\hline$W$ & Width of construction site, in the horizontal $x$ direction \\
\hline$B$ & Length of construction site, in the vertical $y$ direction \\
\hline$W f_{i}$ & Width of facility $i$ in the $x$ direction \\
\hline$L f_{i}$ & Length of facility $i$ in the $y$ direction \\
\hline$B C X_{p}^{m}$ & $x$-coordinate of corner $p$ at location $m$ \\
\hline$B C Y_{p}^{m}$ & $y$-coordinate of corner $p$ at location $m$ \\
\hline$D_{p q}^{m n}$ & $\begin{array}{l}\text { Distance between corners } p \text { and } q \text { located at locations } m \\
\text { and } n \text { respectively }\end{array}$ \\
\hline$C L X_{m}$ & $x$-coordinate of centroid of location $m$ \\
\hline$C L Y_{m}$ & $y$-coordinate of centroid of location $m$ \\
\hline$W L_{m}$ & Width of location $m$ in the horizontal $x$ direction \\
\hline$L L_{m}$ & Length of location $m$ in the vertical $y$ direction \\
\hline
\end{tabular}

\subsubsection{Objective Function}

The objective function (1) consists of a frequency parameter defined over facilities $i$ and $j$ and transportation mode $t$. The transportation mode can involve plants such as trucks, mixers etc. The frequency parameter is multiplied by a distance variable, which is defined by the distance constraints, to be discussed in the next section.

Minimise $\sum_{t} \sum_{i} \sum_{j} F_{i j}^{t} d_{i j}$
Table 3 Variable notation

\begin{tabular}{ll}
\hline Notation & Description \\
\hline$d_{i j}$ & $\begin{array}{l}\text { Distance between facility } i \text { and } j, \\
\text { such that } i \neq j \\
\text { Short Manhattan distance between } \\
\text { facilities } i \text { and } j \text { positioned at } \\
\text { locations deemed close to one } \\
\text { another, such that } i \neq j\end{array}$ \\
$d_{i j}^{s}$ & $\begin{array}{l}\text { Long distance between facilities } i \\
\text { and } j \text { positioned at location deemed } \\
\text { far from one another, such that } i \neq j\end{array}$ \\
$d_{i j}^{m n}$ & $\begin{array}{l}x \text {-coordinate of centroid of facility } i \\
c_{i}^{x}\end{array}$ \\
$c_{i}^{y}$ & $\begin{array}{l}\text { y-coordinate of centroid of facility } i \\
\tau_{i j}^{x 1} \in\{0,1\} \forall i, j \in F_{T}: i \neq j\end{array}$ \\
& $\begin{array}{l}\text { Equals one if } c_{i}^{x} \text { is less than the left } \\
\text { border of facility } j\end{array}$ \\
$\tau_{i j}^{x 2} \in\{0,1\} \forall i, j \in F_{T}: i \neq j$ & $\begin{array}{l}\text { Equals one if } c_{i}^{x} \text { is greater than the } \\
\text { right border of facility } j\end{array}$ \\
$\tau_{i j}^{y 1} \in\{0,1\} \forall i, j \in F_{T}: i \neq j$ & $\begin{array}{l}\text { Equals one if } c_{i}^{y} \text { is less than the } \\
\text { bottom border of facility } j\end{array}$ \\
$\tau_{i j}^{y 2} \in\{0,1\} \forall i, j \in F_{T}: i \neq j$ & $\begin{array}{l}\text { Equals one if } c_{i}^{y} \text { is greater than the } \\
\text { top border of facility } j \\
\mu_{i j}^{x} \in\{0,1\} \forall i, j \in F_{T}: i \neq j\end{array}$ \\
$\mu_{i j}^{y} \in\{0,1\} \forall i, j \in F_{T}: i \neq j$ & $\begin{array}{l}\text { Equals one if facility } i \text { and } j \text { do not } \\
\text { overlap in the horizontal } x \text { direction } \\
\text { overlap in the vertical } y \text { direction. }\end{array}$ \\
\hline &
\end{tabular}

\subsubsection{Distance constraints}

The allocation of facilities to predefined locations decides the method by which distance is measured between facilities. For close-by facilities constraint (2) is deployed where the variable $d_{i j}^{s}$ is defined through constraint (4) as the absolute value distance between the facilities. For long distances, constraints (3) and (5) are applicable where distance is defined in terms of building area corners through which moving plants are required to pass in order to get to the final facility destination. The definition of corners $p$ and $q$ associated with locations $m$ and $n$ respectively, help in delineating an appropriate travel path.

$$
\begin{aligned}
& d_{i j}=\sum_{m \in L} \sum_{n \in L} z_{i m} z_{j n} d_{i j}^{s} \quad \forall i, j \in F: i \neq j, \delta_{m n}=0 \\
& d_{i j}=\sum_{m \in L} \sum_{n \in L} z_{i, m} z_{j, n} d_{i j}^{m n} \quad \forall i, j \in F: i \neq j, \delta_{m n}=1
\end{aligned}
$$




$$
\begin{gathered}
d_{i j}^{s}=\left|c_{i}^{x}-c_{j}^{x}\right|+\left|c_{i}^{y}-c_{j}^{y}\right| \quad \forall i, j \in F: i \neq j \\
d_{i j}^{m n}=\min _{\substack{p \in C_{m} \\
q \in C_{n}}}\left\{\left|c_{i}^{x}-B C X_{p}^{m}\right|+\left|c_{i}^{y}-B C Y_{p}^{m}\right|+\left|c_{j}^{x}-B C X_{q}^{n}\right|+\left|c_{j}^{y}-B C Y_{q}^{n}\right|+D_{p, q}^{m, n}\right\} \\
\forall i, j \in F: i \neq j \quad \forall m, n \in L: \delta_{m n}=1
\end{gathered}
$$

\subsubsection{Allocation constraints}

To ensure that each facility gets positioned in an appropriate space within the construction site, it is necessary to incorporate constraints (6)-(8). Constraint (6) states that for each temporary facility one space has to be allocated. Constraint (7) outlines the possibility of each space handling more than a single facility, while constraint (8) defines the positions of permanent facilities, through the matrix $\lambda_{\text {im }}$

$$
\begin{array}{ll}
\sum_{m \in L} z_{i m}=1 & \forall i \in F_{T} \subseteq F \\
\sum_{i \in F_{T}} z_{i m} \geq 1 & \forall m \in L \\
z_{i m}=1 & \forall(i, m) \in V \mid \lambda_{i m}=1
\end{array}
$$

\subsubsection{Design constraints}

\subsubsection{Non-overlap constraints}

Constraints (9)-(11) define a feasible configuration in which either horizontal or vertical overlap between facilities is allowed, but not both. In particular constraint (9) controls the horizontal overlap while constraint (10) determines whether overlap is present vertically. The presence of constraint (11) ensures that only one of constraints (9) or (10) is active at any one time.

$$
\begin{aligned}
& \left|c_{i}^{x}-c_{j}^{x}\right| \geq 0.5\left(W f_{i}+W f_{j}\right) \cdot \mu_{i j}^{x} \quad \forall i, j \in F_{T}: i \neq j \\
& \left|c_{i}^{y}-c_{j}^{y}\right| \geq 0.5\left(L f_{i}+L f_{j}\right) \cdot \mu_{i j}^{y} \quad \forall i, j \in F_{T}: i \neq j \\
& 1-z_{i m} z_{j n}+\mu_{i j}^{x}+\mu_{i j}^{y} \geq 1 \quad \forall i, j \in F: i \neq j \forall m, n \in L: m=n
\end{aligned}
$$

\subsubsection{Boundary constraints}

Facilities are required to be located within the boundaries demarcated by the location to which they have been assigned. As such, constraints (12)-(15) are initiated. Constraints (12) and (13) force the facility to lie in between the vertical boundaries, while constraints (14) and (15) require the facility to be positioned within the horizontal boundaries.

$$
\begin{aligned}
& c x_{i}+\left(0.5 W f_{i}\right) \leq\left(C L X_{m}+0.5 W L_{m}\right) z_{\text {iim }}+W\left(1-z_{\text {im }}\right) \quad \forall i \in F \quad \forall m \in L \\
& c x_{i}-\left(0.5 W f_{i}\right) \geq\left(C L X_{m}-0.5 W L_{m}\right) z_{\text {im }} \quad \forall i \in F \quad \forall m \in L \\
& c y_{i}+\left(0.5 L f_{i}\right) \leq\left(C L Y_{m}+0.5 L L_{m}\right) z_{\text {im }}+B\left(1-z_{\text {iim }}\right) \quad \forall i \in F \quad \forall m \in L \\
& c y_{i}-\left(0.5 L f_{i}\right) \geq\left(C L Y_{m}-0.5 L L_{m}\right) z_{\text {im }} \quad \forall i \in F \quad \forall m \in L
\end{aligned}
$$

\subsubsection{Travel interference constraints}

To prevent collision into already allocated facilities during movements across the construction site, constraints (16)-(20) are effectuated. For there to be no vertical travel interference between two facilities at the same location, constraints (16) and (17) are deployed. It is also a requirement to prevent travel interference in the horizontal direction, and this is specified by constraints (18) and (19). To ensure that horizontal and vertical travel non- interference ensues at any one time, constraints (20) and (21) need to be activated.

$$
\begin{array}{ll}
c_{i}^{x} \leq c_{j}^{x}-0.5 W f_{j}+W\left(1-\tau_{i j}^{x 1}\right) & \forall i, j \in F_{T}: i \neq j \\
c_{i}^{x} \geq c_{j}^{x}+0.5 W f_{j}-W\left(1-\tau_{i j}^{x 2}\right) & \forall i, j \in F_{T}: i \neq j \\
c_{i}^{y} \leq c_{j}^{y}-0.5 L f_{j}+B\left(1-\tau_{i j}^{y 1}\right) & \forall i, j \in F_{T}: i \neq j \\
c_{i}^{y} \geq c_{j}^{y}+0.5 L f_{j}-B\left(1-\tau_{i j}^{y 2}\right) & \forall i, j \in F_{T}: i \neq j \\
1-z_{i i n} z_{j n}+\tau_{i j}^{x 1}+\tau_{i j}^{x 2} \geq 1 \quad \forall i, j \in F_{T}: i \neq j \forall m, n \in L: m=n \\
1-z_{i i n} z_{j n}+\tau_{i j}^{y 1}+\tau_{i j}^{y 2} \geq 1 \quad \forall i, j \in F_{T}: i \neq j \forall m, n \in L: m=n
\end{array}
$$

\subsubsection{Domain of variables}

The final set of constraints, represented by constraints (22)-(26) are put in place to define the range of values which the variables involved in the model can take. All variables apart from $z_{i m}$ which are defined as integer variables, as presented in table 3 , are assumed to be positive and continuous.

$$
\begin{array}{ll}
c_{i}^{x} \geq 0 & \forall i \in F \\
c_{i}^{y} \geq 0 & \forall i \in F \\
d_{i j} \geq 0 & \forall i, j \in F: i \neq j \\
d_{i j}^{s} \geq 0 & \forall i, j \in F: i \neq j \\
d_{i j}^{m n} \geq 0 & \forall i, j \in F: i \neq j \quad \forall m, n \in L: \delta_{m n}=1
\end{array}
$$

Model F-SLP is therefore represented by equations (1)(26). 


\subsection{O-SLP: Open Region SLP}

Model O-SLP involves an adjustment to objective function (1) embedded in the chief model. Here, the distance measure between facilities is assumed to follow a Manhattan approach, whereby direct rectilinear routes are assumed between the centroids of the facilities. The resulting objective function is displayed in equation (27). The design constraints used in the previously explicated model are still applicable to Model O-SLP ((6)-(25)), except for the distance constraints ((2)-(5)), where corner points to avoid the forbidden region, this being the projects footprint construction zone, is neglected.

The distance measure implemented for the variable $d_{i j}^{s}$, as is shown in equation (28), is in a purely rectilinear form.

$$
\begin{aligned}
& \text { Minimise } \sum_{t} \sum_{i} \sum_{j} F_{i j}^{t} d_{i j}^{s} \\
& d_{i j}^{s}=\left|c_{i}^{x}-c_{j}^{x}\right|+\left|c_{i}^{y}-c_{j}^{y}\right| \quad \forall i, j \in F: i \neq j
\end{aligned}
$$

Model O-SLP is thus represented by equations (6-25) and equations (27) and (28).

\subsection{R-SLP: Relaxed SLP}

A relaxed model is presented where the SLP problem is solved in two stages. In the first stage the facilities are optimised across all predefined locations, with only a single set of constraints. The distance between the locations, $D_{m n}$, is assumed to be given. The objective function and the associated constraints are defined by equations (29) and (30), respectively. No other constraints are included in the first stage. The model therefore initially solves for the location variables $z_{i m}$ and $z_{j n}$

$$
\begin{aligned}
& \text { Minimise } \sum_{t} \sum_{i} \sum_{j} F_{i j}^{t} d_{i j} \\
& d_{i j}=\sum_{n \in L} \sum_{m \in L} z_{i m} z_{j n} D_{m n} \quad \forall i, j \in F: i \neq j
\end{aligned}
$$

Once the first stage of the optimisation is complete, and all facilities have been allocated to their optimised positions, the second stage is initiated. This involves the implementation of $|L|$ sub-optimisation problems where a model, termed FL(m) for each location $m$ is solved such that design constraints (i.e. non-overlap constraints, the boundary constraints and the travel interference constraints) are applied. A solution, labelled $z_{\text {im }}^{*}$, is generated once the first stage is solved. Cuts, defined by equations (31) and (32) are then introduced, in the form of additional constraints added to the model, depending on the feasibility of each sub-problem. The cuts introduced take the form of specifying which combinations of the facilities are forbidden, assigned by $z_{i m}^{*}$, at location $m$ where the design constraints are violated. This process is repeated until a globally feasible configuration is obtained. The Pseudo code of this constraint generation algorithm is displayed in Figure 1.

$$
\begin{aligned}
& \sum_{i \in F_{T}: z_{i m}^{*}=1} z_{i m} \leq N_{m}-1 \quad \forall m \in L \\
& N_{m}=\sum_{i \in F_{T}} z_{i m}^{*} \quad \forall m \in L
\end{aligned}
$$

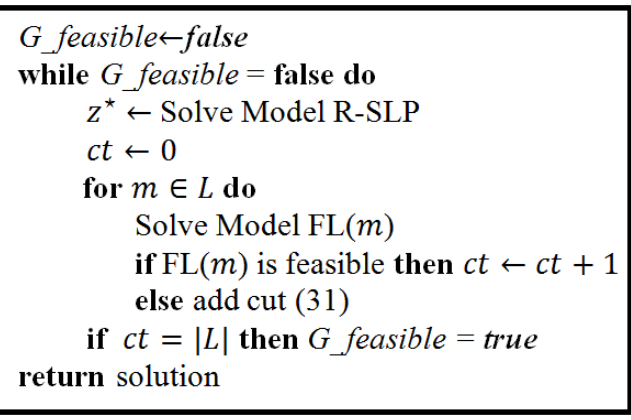

Figure 1. Pseudo code of constraint generation algorithm developed for Model R-SLP

\section{Application and Numerical Results}

To establish a relative ground for comparing the models presented in this paper, a hypothetical case example is employed. The case study represents a construction site with a project comprising of a large shopping centre. A general purpose solver, namely CPLEX, is used to solve all three models [37]. A single type of solver is adopted to ensure a fair comparison between the three models. The shopping centre building footprint, along with the predefined locations within the construction site are displayed in Figure 2.

The comparison was carried out on the basis of computational time and the value of the obtained optimum solution from each model. The final solution garnered from the model signifies the total monetary cost of moving material around the construction site, measured in Australian dollars. Table 4 displays the facilities to be allocated along with their dimensions. The travel frequency parameters, assumed between the facilities, are presented in Tables 5-7. Three frequency tables are shown since it is assumed that three different transportation modes are deployed to move materials 
between facilities. Only on-ground transportation is considered, as it is presumed that the materials are unloaded at depot areas located outside the construction project area footprint, from which aerial transportation via tower cranes is then employed.

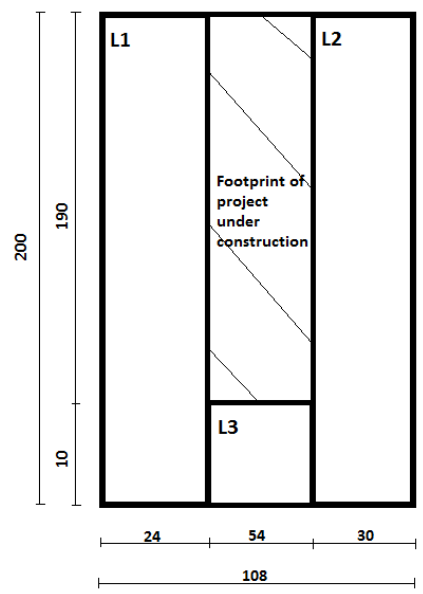

Figure 2. Construction site dimensions

Figure 3 is a graph of the computational time spent in reaching the optimum solution by each of the three models. It is clear that Model F-SLP, where a computational time of 1330 seconds is recorded, takes longer in comparison with the rest models. The other two models were much quicker having recorded computational times of 86 seconds and 162 seconds for Model O-SLP and Model R-SLP, respectively. The quickest model to produce results was therefore Model O-SLP. If these results are weighed with those displayed in Figure 4, where the optimum solution values obtained are depicted, then it becomes evident that Model F-SLP is the one that fairs best in terms of the solution value obtained but not in terms of the computational time required to achieve it. That is because Model F-SLP contains many details yet is the model that produces the global optimum solution. By adopting the objective function of Model F-SLP as the evaluating point for solution values obtained by the different models, Model O-SLP is found to perform well when computational time and optimum solution value are considered since when compared against the solution of Model F-SLP, a $1.5 \%$ difference in cost value is observed, with Model O-SLP producing a site layout configuration carrying a cost of $\$ 29012$, whereas Model F-SLP results in a cost of \$28583. Model R-SLP outputs a value of $\$ 30141$, generating the highest cost site layout configuration, with a deviation of $5.4 \%$ from the global optimum solution derived from Model F-SLP. It is worth noting that the solution generated by Model R-SLP is likely to be sub-optimal due to the dissection of the optimisation process into stages.

The results obtained from Models O-SLP and R-SLP, where the minor differences in monetary costs of the resulting facility configurations, and the speed at which such solutions were fashioned, highlight the potential of producing reasonable results by a relaxed model in shorter time periods. The results of the site layout configurations generated from each of the three models are shown in Table 8.

Table 4. Temporary Facilities

\begin{tabular}{cccc}
\hline Facility & Symbol & $\begin{array}{c}\text { Width in } x \text { - } \\
\text { direction } \\
(\mathrm{m})\end{array}$ & $\begin{array}{c}\text { Length in } y \text { - } \\
\text { direction } \\
(\mathrm{m})\end{array}$ \\
\hline Steel Yard & F1 & 8 & 8 \\
Formwork Assembly & F2 & 8 & 8 \\
Concrete Batch Plant & F3 & 8 & 8 \\
False Work & F4 & 8 & 8 \\
Offices & F5 & 8 & 8 \\
Warehouse & F6 & 8 & 8 \\
Generator Room & F7 & 8 & 8 \\
\hline
\end{tabular}

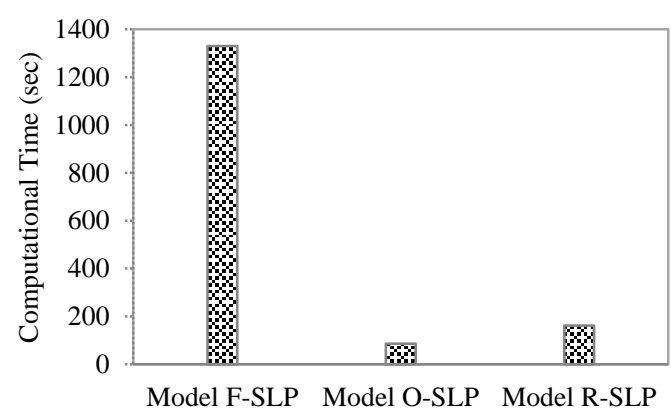

Figure 3. Computational performance of the three models

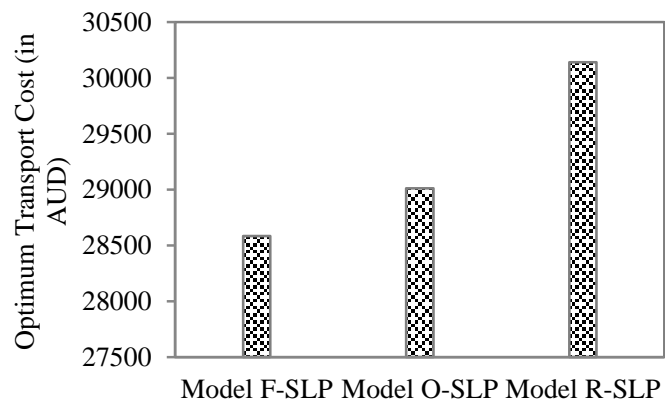

Figure 4. Optimum transport costs obtained by the three models. 
Table 5. Frequency of travel of Forklift Truck between facilities

\begin{tabular}{|c|c|c|c|c|c|c|c|c|c|c|c|}
\hline \multirow{2}{*}{ Facility $i$} & \multicolumn{11}{|c|}{ Facility $j$} \\
\hline & F1 & $\mathrm{F} 2$ & F3 & F4 & F5 & F6 & F7 & F8 & F9 & F10 & F11 \\
\hline F1 & 0 & 7000 & 200 & 4500 & 20 & 13000 & 10 & 24000 & 24000 & 24000 & 24000 \\
\hline $\mathrm{F} 2$ & 7000 & 0 & 1000 & 2000 & 20 & 8900 & 10 & 14000 & 14000 & 14000 & 14000 \\
\hline F3 & 200 & 1000 & 0 & 30 & 20 & 10000 & 10 & 50 & 50 & 50 & 50 \\
\hline F4 & 4500 & 2000 & 30 & 0 & 20 & 7500 & 10 & 11000 & 11000 & 11000 & 11000 \\
\hline F5 & 20 & 20 & 20 & 20 & 0 & 10 & 10 & 10 & 10 & 10 & 10 \\
\hline F6 & 13000 & 8900 & 10000 & 7500 & 10 & 0 & 10 & 9000 & 9000 & 9000 & 9000 \\
\hline F7 & 10 & 10 & 10 & 10 & 10 & 10 & 0 & 10 & 10 & 10 & 10 \\
\hline F8 & 24000 & 14000 & 50 & 11000 & 10 & 9000 & 10 & 0 & 4000 & 4000 & 4000 \\
\hline F9 & 24000 & 14000 & 50 & 11000 & 10 & 9000 & 10 & 4000 & 0 & 4000 & 4000 \\
\hline F10 & 24000 & 14000 & 50 & 11000 & 10 & 9000 & 10 & 4000 & 4000 & 0 & 4000 \\
\hline F11 & 24000 & 14000 & 50 & 11000 & 10 & 9000 & 10 & 4000 & 4000 & 4000 & 0 \\
\hline
\end{tabular}

Table 6. Frequency of travel of Dropside Truck between facilities

\begin{tabular}{cccccccccccc}
\hline \multirow{2}{*}{ Facility $i$} & \multicolumn{10}{c}{ Facility $j$} \\
\cline { 2 - 12 } & F1 & F2 & F3 & F4 & F5 & F6 & F7 & F8 & F9 & F10 & F11 \\
\hline F1 & 0 & 40200 & 300 & 20000 & 10 & 33000 & 10 & 6000 & 6000 & 6000 & 6000 \\
F2 & 40200 & 0 & 370 & 23000 & 10 & 26700 & 10 & 4700 & 4700 & 4700 & 4700 \\
F3 & 300 & 370 & 0 & 50 & 10 & 30000 & 10 & 75 & 75 & 75 & 75 \\
F4 & 20000 & 23000 & 50 & 0 & 10 & 18000 & 10 & 3500 & 3500 & 3500 & 3500 \\
F5 & 10 & 10 & 10 & 10 & 0 & 10 & 10 & 10 & 10 & 10 & 10 \\
F6 & 33000 & 26700 & 30000 & 18000 & 10 & 0 & 10 & 900 & 900 & 900 & 900 \\
F7 & 10 & 10 & 10 & 10 & 10 & 10 & 0 & 10 & 10 & 10 & 10 \\
F8 & 6000 & 4700 & 75 & 3500 & 10 & 900 & 10 & 0 & 250 & 250 & 250 \\
F9 & 6000 & 4700 & 75 & 3500 & 10 & 900 & 10 & 250 & 0 & 250 & 250 \\
F10 & 6000 & 4700 & 75 & 3500 & 10 & 900 & 10 & 250 & 250 & 0 & 250 \\
F11 & 6000 & 4700 & 75 & 3500 & 10 & 900 & 10 & 250 & 250 & 250 & 0 \\
\hline
\end{tabular}

Table 7. Frequency of travel of Cement Mixer Truck between facilities

\begin{tabular}{cccccccccccc}
\hline \multirow{2}{*}{ Facility $i$} & \multicolumn{10}{c}{ Facility $j$} \\
\cline { 2 - 12 } & F1 & F2 & F3 & F4 & F5 & F6 & F7 & F8 & F9 & F10 & F11 \\
\hline F1 & 0 & 10 & 10 & 10 & 10 & 10 & 10 & 10 & 10 & 10 & 10 \\
F2 & 10 & 0 & 10 & 10 & 10 & 10 & 10 & 10 & 10 & 10 & 10 \\
F3 & 10 & 10 & 0 & 0 & 10 & 10 & 0 & 3800 & 3800 & 3800 & 3800 \\
F4 & 10 & 10 & 0 & 0 & 10 & 10 & 10 & 10 & 10 & 10 & 10 \\
F5 & 10 & 10 & 10 & 10 & 0 & 10 & 10 & 10 & 10 & 10 & 10 \\
F6 & 10 & 10 & 10 & 10 & 10 & 0 & 10 & 10 & 10 & 10 & 10 \\
F7 & 10 & 10 & 0 & 10 & 10 & 10 & 0 & 10 & 10 & 10 & 10 \\
F8 & 10 & 10 & 3800 & 10 & 10 & 10 & 10 & 0 & 10 & 10 & 10 \\
F9 & 10 & 10 & 3800 & 10 & 10 & 10 & 10 & 10 & 0 & 10 & 10 \\
F10 & 10 & 10 & 3800 & 10 & 10 & 10 & 10 & 10 & 10 & 0 & 10 \\
F11 & 10 & 10 & 3800 & 10 & 10 & 10 & 10 & 10 & 10 & 10 & 0 \\
\hline
\end{tabular}


Table 8. Optimised locations of temporary facilities

\begin{tabular}{ccccccc}
\hline & \multicolumn{2}{c}{ Model 1 } & \multicolumn{2}{c}{ Model 2 } & \multicolumn{2}{c}{ Model 3 } \\
\cline { 2 - 7 } Facility & $\mathrm{x}$ & $\mathrm{y}$ & $\mathrm{x}$ & $\mathrm{y}$ & $\mathrm{x}$ & $\mathrm{y}$ \\
\cline { 2 - 7 } F1 & 28 & 6 & 37.5 & 6 & 48 & 62 \\
F2 & 20 & 6 & 20 & 6 & 73 & 80 \\
F3 & 4 & 22 & 4 & 22 & 3 & 100 \\
F4 & 82 & 10 & 82 & 11 & 53 & 4 \\
F5 & 90 & 18 & 90 & 19 & 103 & 40 \\
F6 & 12 & 14 & 12 & 14 & 83 & 50 \\
F7 & 98 & 26 & 98 & 27 & 5 & 82 \\
\hline
\end{tabular}

\section{Conclusion}

Given the results presented in this paper, it can be concluded that the format in which the SLP model is formulated does have an effect on the overall optimisation results, both in terms of the optimum solution obtained and on the computational time required to achieve such a solution. Even though it may be desirable to model extra details in terms of the route path followed on a construction site, accurate representations will require greater computational times. At the same time, developing relaxation models seems a viable option particularly when quick results are needed, for a thorough sensitivity analysis to be conducted. Future work will involve the development of more representations that assess the impacts of model formulations on instance difficulty of the SLP problem.

\section{References}

[1] A. Hamiani, "CONSITE: a knowledge-based expert system framework for construction site layout," Texas Univ., Austin (USA), Jan. 1987.

[2] A. Drira, H. Pierreval, and S. Hajri-Gabouj, "Facility layout problems: A survey," Annu. Rev. Control, vol. 31, no. 2, pp. 255-267, 2007.

[3] T. Koopmans and M. J. Beckmann, "Assignment Problems and the Location of Economic Activities," Cowles Foundation for Research in Economics, Yale University, 4, 1955.

[4] T. Hegazy and E. Elbeltagi, "EvoSite: EvolutionBased Model for Site Layout Planning," J. Comput. Civ. Eng., vol. 13, no. 3, pp. 198-206, 1999.

[5] P. P. Zouein, MoveSchedule: a planning tool for scheduling space use on construction sites. 1996.

[6] M. Andayesh and F. Sadeghpour, "The time dimension in site layout planning," Autom. Constr., vol. 44, pp. 129-139, Aug. 2014.

[7] E. Elbeltagi, T. Hegazy, and A. Eldosouky, "Dynamic Layout of Construction Temporary Facilities Considering Safety," J. Constr. Eng. Manag., vol. 130, no. 4, pp. 534-541, 2004.

[8] H. Said and K. El-Rayes, "Performance of global optimization models for dynamic site layout planning of construction projects," Autom. Constr., vol. 36, pp. 71-78, Dec. 2013.

[9] H. Sanad, M. Ammar, and M. Ibrahim, "Optimal Construction Site Layout Considering Safety and Environmental Aspects," J. Constr. Eng. Manag., vol. 134, no. 7, pp. 536-544, 2008.

[10] I. D. Tommelein and P. P. Zouein, "Activity-level space scheduling," ISARC Proc., 1992.

[11] F. Zhou, S. M. AbouRizk, and H. AL-Battaineh, "Optimisation of construction site layout using a hybrid simulation-based system," Simul. Model. Pract. Theory, vol. 17, no. 2, pp. 348-363, Feb. 2009.

[12] P. Zouein and I. Tommelein, "Dynamic Layout Planning Using a Hybrid Incremental Solution Method," J. Constr. Eng. Manag., vol. 125, no. 6, pp. 400-408, 1999.

[13] A. W. A. Hammad, D. Rey, and A. Akbarnezhad, "A mixed-integer nonlinear programming model for minimising construction site noise levels through site layout optimisation," presented at the 31st International Symposium on Automation and Robotics in Construction and Mining, ISARC 2014 - Proceedings, 2014, pp. 722-729.

[14] K. El-Rayes and A. Khalafallah, "Trade-off between Safety and Cost in Planning Construction Site Layouts," J. Constr. Eng. Manag., vol. 131, no. 11, pp. 1186-1195, 2005.

[15] A. Khalafallah and K. El-Rayes, "Automated multi-objective optimization system for airport site layouts," Autom. Constr., vol. 20, no. 4, pp. 313-320, Jul. 2011.

[16] A. Khalafallah and K. El-Rayes, "Minimizing Construction-Related Security Risks during Airport Expansion Projects," J. Constr. Eng. Manag., vol. 134, no. 1, pp. 40-48, 2008.

[17] X. Ning, K.-C. Lam, and M. C.-K. Lam, "A decision-making system for construction site layout planning," Autom. Constr., pp. 459-473, Jul. 2011

[18] J. Xu and Z. Li, "Multi-Objective Dynamic Construction Site Layout Planning in Fuzzy Random Environment," Autom. Constr., vol. 27, pp. 155-169, Nov. 2012

[19] S. Zhao and Z. Li, "Multi-objective Optimization for Construction Site Layout Planning Problem under Fuzzy Random Environment," in 2014 Seventh International Joint Conference on Computational Sciences and Optimization (CSO), 2014, pp. 641-645.

[20] A. M. Adrian, A. Utamima, and K.-J. Wang, "A comparative study of GA, PSO and ACO for solving construction site layout optimization," KSCE J. Civ. Eng., pp. 1-8, Sep. 2014. 
[21] M. Andayesh and F. Sadeghpour, "Dynamic site layout planning through minimization of total potential energy," Autom. Constr., vol. 31, pp. 92-102, May 2013.

[22] B. Kalmár, A. Kalmár, K. Balázs, and L. T. Kóczy, "Construction Site Layout and Building Material Distribution Planning Using Hybrid Algorithms," in Issues and Challenges of Intelligent Systems and Computational Intelligence, L. T. Kóczy, C. R. Pozna, and J. Kacprzyk, Eds. Springer International Publishing, 2014, pp. 75-88.

[23] K. Lam, X. Ning, and T. Ng, "The application of the ant colony optimization algorithm to the construction site layout planning problem," Constr. Manag. Econ., vol. 25, no. 4, pp. 359-374, 2007.

[24] H. Li and P. E. . Love, "Genetic search for solving construction site-level unequal-area facility layout problems," Autom. Constr., vol. 9, no. 2, pp. 217-226, Mar. 2000.

[25] M. Mawdesley, Al-jibouri S., and H. Yang, "Genetic Algorithms for Construction Site Layout in Project Planning," J. Constr. Eng. Manag., vol. 128, no. 5, pp. 418-426, 2002.

[26] M. Yahya and M. P. Saka, "Construction site layout planning using multi-objective artificial bee colony algorithm with Levy flights," Autom. Constr., vol. 38, pp. 14-29, Mar. 2014.

[27] S. Easa and K. Hossain, "New Mathematical Optimization Model for Construction Site Layout," J. Constr. Eng. Manag., vol. 134, no. 8, pp. 653662, 2008.

[28] C. Wong, I. Fung, and C. Tam, "Comparison of Using Mixed-Integer Programming and Genetic Algorithms for Construction Site Facility Layout Planning," J. Constr. Eng. Manag., vol. 136, no. 10, pp. 1116-1128, 2010 .

[29] P. Zouein, H. Harmanani, and A. Hajar, "Genetic Algorithm for Solving Site Layout Problem with Unequal-Size and Constrained Facilities," $J$. Comput. Civ. Eng., vol. 16, no. 2, pp. 143-151, 2002.

[30] C. Huang, C. K. Wong, and C. M. Tam, "Optimization of tower crane and material supply locations in a high-rise building site by mixedinteger linear programming," Autom. Constr., vol. 20, no. 5, pp. 571-580, Aug. 2011.

[31] H. Li and P. Love, "Site-Level Facilities Layout Using Genetic Algorithms," J. Comput. Civ. Eng., vol. 12, no. 4, pp. 227-231, 1998.

[32] K. C. Lam, C. M. Tang, and W. C. Lee, "Application of the entropy technique and genetic algorithms to construction site layout planning of medium-size projects," Constr. Manag. Econ., vol. 23, no. 2, pp. 127-145, Feb. 2005.

[33] J. Xu and X. Song, "Suggestions for Temporary Construction Facilities' Layout Problems in Large-Scale Construction Projects," J. Constr. Eng. Manag., vol. 140, no. 5, p. 06014001, 2014.

[34] K. Smith-Miles and L. Lopes, "Measuring instance difficulty for combinatorial optimization problems," Comput. Oper. Res., vol. 39, no. 5, pp. 875-889, May 2012.

[35] C. A. Floudas, Nonlinear and Mixed-Integer Optimization: Fundamentals and Applications: Fundamentals and Applications. Oxford University Press, 1995.

[36] L. Liberti, S. Cafieri, and F. Tarissan, "Reformulations in Mathematical Programming: A Computational Approach," in Foundations of Computational Intelligence Volume 3, A. Abraham, A.-E. Hassanien, P. Siarry, and A. Engelbrecht, Eds. Springer Berlin Heidelberg, 2009, pp. 153-234.

[37] "IBM CPLEX Optimizer - United States," 30Dec-2014. [Online]. Available: http://www01.ibm.com/software/commerce/optimization/cpl ex-optimizer/. [Accessed: 05-Feb-2015]. 